\title{
Wide Tuning Range CMOS VCO
}

\author{
Abhishek Kumar ${ }^{\mathrm{a}^{*}}$ and Kriti Tiwari ${ }^{\mathrm{b}}$ \\ ${ }^{a}$ Assistant Professor, Lovely Professional University, Punjab, India \\ ${ }^{b}$ Student, Lovely Professional University, Punjab, India
}

Received: 14 January 2017; Accepted: 13 May 2017; Published: 08 September 2017

\begin{abstract}
A communication system requires a highly stabilized frequency; LC and RC oscillator are two wide option for frequency generation. Performance of an LC oscillator suffers from leakage, area, noise etc compare RC oscillator. Voltage controlled oscillator (VCO) is preferred category if RC oscillator to generate high oscillator frequency. VCO contains odd number of delay stage cascaded together; output frequency strongly depends on switching threshold of individual stages. A 3 stage current starved VCO can generate upto $0.6 \mathrm{Ghz}$; this work is focused around body bias technique to increase frequency without increasing number of delay stage. Oscillation frequency has been controlled by bulk terminal of PMOS and NMOS individually and by means of adaptive body bias network. Cadence spectre based simulation result at CMOS 90nm shows that by reverse biasing of PMOS obtained frequency is $1.2 \mathrm{GHz}-10.01 \mathrm{GHz}$ with tuning range of $95 \%$ while biasing of NMOS generate frequency of $500 \mathrm{MHz}-12.5 \mathrm{GHz}$ with tuning range capability of $96 \%$. This design presents high frequency with wider tuning range and optimum reduced power by which this oscillator design can be used in different band of communication.
\end{abstract}

Index Terms: Ring oscillators, Voltage-controlled oscillators, Frequency, Phase noise, Tuning, Bandwidth.

(C) 2017 Published by MECS Publisher. Selection and/or peer review under responsibility of the Research Association of Modern Education and Computer Science.

\section{Introduction}

Voltage controlled oscillator is commonly investigated as it is a crucial circuit of phase locked loop (PLL), clock and data recovery circuits (CDR), Radio Frequency application. Ring oscillator is the primary component of VCO; a cascade combination of delay stages connected as closed loop. In satellite / microwave communication clock data recovery unit at receiver restore the clock from received data with the help of PLL $[1,2]$. Ring oscillator is the heart of VCO it generates oscillating frequency in specific band (up to $30 \mathrm{GHz}$ ). In

* Corresponding author. Tel.: 9888431215

E-mail address: abhishek.15393@lpu.co.in 
this work current starved topology has been consider as basic element for delay calculation [14]; it limits the current to the inverter for charging and discharging of load there by controlling the stage delay and oscillation frequency. Current starved ring oscillator can generate frequency $4 \mathrm{GHz}$ applicable for $\mathrm{L}, \mathrm{S}$ and $\mathrm{C}$-band. In this work frequency range tried improve up to $12.5 \mathrm{GHz}$ for K-band application using body biasing technique. Body biasing of MOS effectively alter the threshold voltage it significantly improves the delay stage result in high frequency.

A current starved topology shown in Fig1, it is observed that MOSFETs M2 and M3 operate as an inverter, while MOSFETs M1 and M4 operate as current sources. The main control on the inverter chain is of $V_{\text {invco }}$ to modify the current that flow from the N1 and P1. The current from these transistors due to direct connection of M5 with M1 along with following transistor and M6 with M4 followed by transistor in series mirror the current in each. Change in the control voltage induces by change in inverter current. $V_{\text {invco modulates the turn on }}$ resistance of pull down transistor M1 and through the current mirror, the pull-up transistor M6 [3-7]. Large value of $\mathrm{V}_{\text {invco }}$ allows a large current to flow, producing a small resistance and a small delay. Cadence Spetre based simulation justify that a current starved ring oscillator can produce frequency $626 \mathrm{MHz}$ at Vdd $1 \mathrm{~V}$.

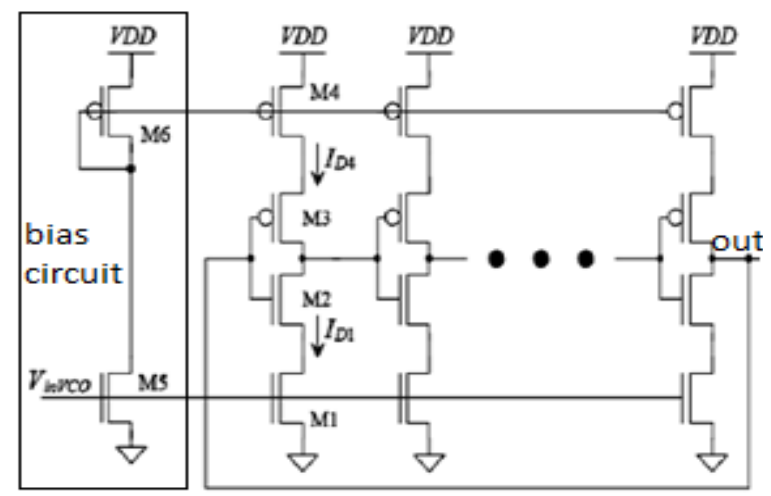

Fig.1. Current Starved Ring Oscillator [8, 9]

Oscillator's frequency primarily depends in threshold voltage of MOS; body biasing is one of technique through which threshold voltage of particular MOS can be varied; usually PMOS body terminal should connect to maximum supply and NMOS body to minimum ground; but additional voltage at body terminal vary the threshold voltage by eqn (1). Threshold voltage of MOS increases by reverse biasing and decrease with forward biasing $[1,2,11]$. Effect of body voltage over represented by, $\gamma$ is body bias factor, Vth decrease for higher $\mathrm{V}_{\mathrm{SB}}$ and decreases for lower $\mathrm{V}_{\mathrm{SB}}$.

$$
V_{T h}=V_{T 0}+\gamma\left(\sqrt{\left|-2 \phi_{F}+V_{S B}\right|}-\sqrt{\left|2 \phi_{F}\right|}\right.
$$

\subsection{Organization of paper}

The second section of the paper elaborate current starve ring oscillator with PMOS and NMOS body bias individually and their effect over frequency and other parameter explored. Section-3 of the paper incorporates conclusion and possible future work.

\section{Current Starve VCO with Body Bias}

A ring oscillator frequency can enhance by an additional supply at body terminal, reverse biasing body 
reduces Vth in case of PMOS and increase in case of Vth. Variation in Vth configure delay of MOS, in this section effect of reverse bias over frequency has been explored.

\subsection{Current Starve Ring Oscillator with PMOS Body Biasing}

Fig2 shows a 3 Stage current starved topology of ring oscillator. In the presented VCO, substrates of PMOS have been connected to dc supply which is kept at optimum value $0.5 \mathrm{~V}$, Vctrl is varied in the range of 0 to $1 \mathrm{~V}$, and Simulations have been carried out using cadence spectre based on CMOS 90nm technology at supply $1 \mathrm{~V}$. PMOS reverse body bias increase threshold voltage and leads to high oscillation frequency. Positive bulk terminal input reduced sub threshold leakage current and minimizes the power dissipation $[8,9,10]$.

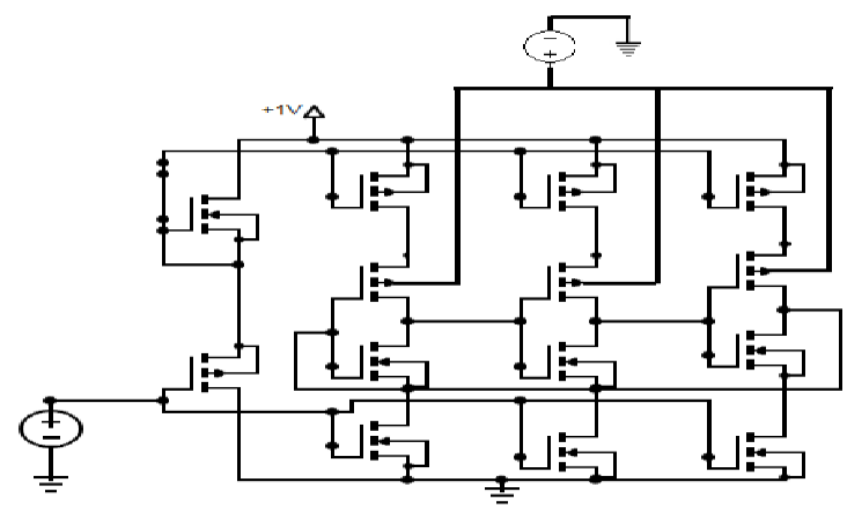

Fig.2. Current Starved Ring Oscillator with PMOS Body Biasing

On current increases with increasing body voltage increases upto $\mathrm{V}_{\mathrm{SB}}=0.5 \mathrm{~V}$. Further increase in the body voltage decrease the value of drain current $\left(\mathrm{I}_{\mathrm{DS}}\right)$. Fig3 shows variation of frequency w.r.t Vctrl keeping body voltage at optimum value $0.5 \mathrm{~V}$. Current starve VCO with PMOS Body Biasing voltage can achieve maximum frequency oscillation frequency 1.2 to $11 \mathrm{GHz}$ while $\mathrm{Vctrl}$ ranges from $0-1 \mathrm{~V}$ at body voltage $\mathrm{V}_{\mathrm{SB}} 0.5 \mathrm{~V}$. Power consumption of the above circuit is $30 \mathrm{uw}$.

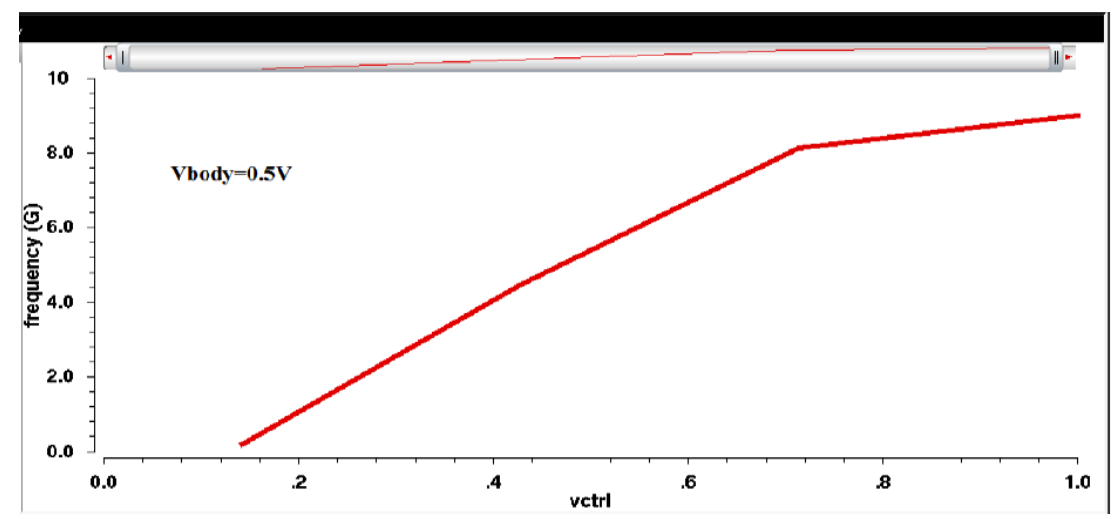

Fig.3. Frequency Variation with Vctrl at Body Voltage 0.5V

\subsection{Current Starve Ring Oscillator with NMOS Body Biasing}


Fig4 present a 3 Stage current starved topology of ring oscillator with substrates of NMOS have been connected to dc supply which is kept at optimum value $0.5 \mathrm{~V}$, Vctrl is varied in the range of $0-1 \mathrm{~V}$. NMOS substrate bias technique with VCO enhance the oscillation frequency $[12,13]$. A positive voltage at the body of NMOS, results on reduction in threshold voltage which leads to high power consumption of the circuit. So it is desirable to choose the optimum value of Vctrl.

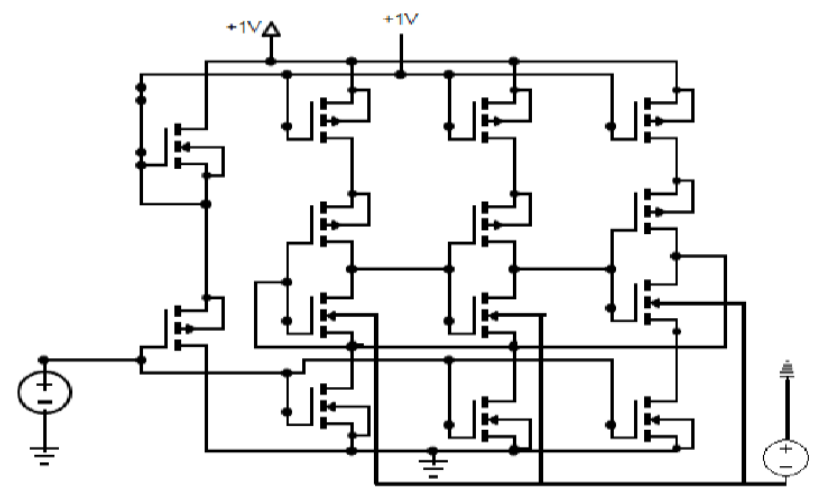

Fig.4. Current Starved ring oscillator with PMOS body biasing

Increasing the body voltage there is increase in the On current up to $\mathrm{V}_{\mathrm{SB}}=0.5 \mathrm{~V}$; further increase in the body voltage decrease the value of $\mathrm{I}_{\mathrm{DS}}$. Fig5 shows variation of frequency w.r.t Vctrl keeping body voltage at optimum value $0.5 \mathrm{~V}$. Current starve VCO with PMOS Body bias increases frequency proportionally 0.5 to $12.5 \mathrm{GHz}$ with control voltage ranges from 0 to $1 \mathrm{~V}$. Power consumption of NMOS body biased current starve $\mathrm{VCO}$ is drastically increases with increases in oscillation frequency. For frequency $12.5 \mathrm{GHz}$ power consumption increases up to $250 \mathrm{uW}$.

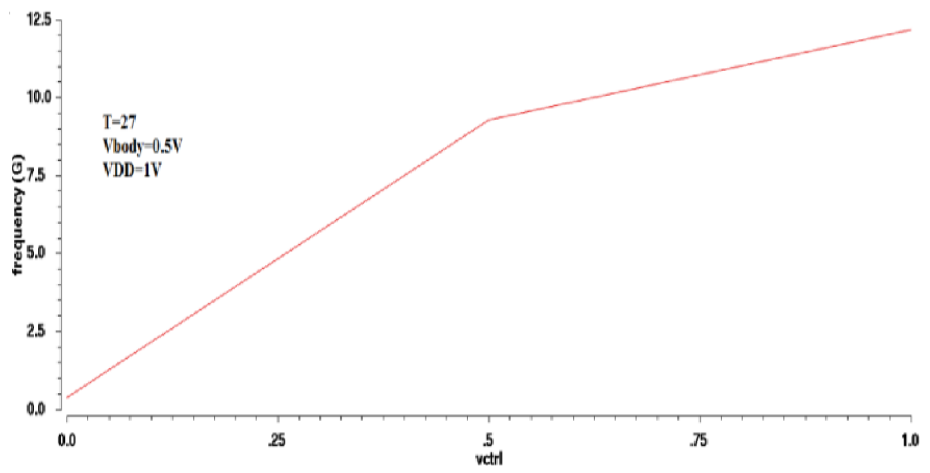

Fig.5. Frequency Variation with Vctrl at body voltage $0.5 \mathrm{~V}$

\subsection{Adaptive Body Bias Ring Oscillators}

Adaptive Body Biasing Ring oscillator is the technique of utilizing the transistor body effect to change transistor threshold voltage during operation by applying adaptive body bias either in forward bias or reverse bias. In this design different body voltages at different Instant of time can be applied which targets to overall wider tuning range of circuit with high frequency along with optimum power saving and phase noise. A fine or 
wider tuning range of frequency is major achievement of this work. The bulk terminal of the oscillator is controlled appropriately depending on the required specifications of the circuit. Objective of adaptive body biasing is to maintain the transistor threshold voltage to retain the device performance by applying either forward body biasing or reverse body biasing. Fig6 presents a current starve ring oscillator with adaptive body bias (ABB) network, ABB can be implemented using multiplexer, select lines S0 and S1; it generates a dc output voltage which bias the body of MOS devices in oscillator circuit. Output obtained from ABB circuit is mentioned in table1. The output voltage level obtained from this circuit maintains bulk of MOS more positive or more negative. The performance of the oscillator is maintained in terms of current driving capability by applying either forward adaptive body biasing or reverse adaptive body biasing. Table1 presents 4 different voltage levels generated by $\mathrm{ABB}$ circuit; Oscillation frequency $(12.54$ to $12.62 \mathrm{GHz})$ is unaffected from $\mathrm{ABB}$ network. Power consumption greatly decreased from 749 uw to $197 \mathrm{uw}$ while bulk supply ranges from $0.745 \mathrm{~V}$ to $66.4 \mathrm{uV}$ attainable amplitude of the designed ring oscillator's frequency is $0.935 \mathrm{~V}$ for $1 \mathrm{~V}$ supply voltage.

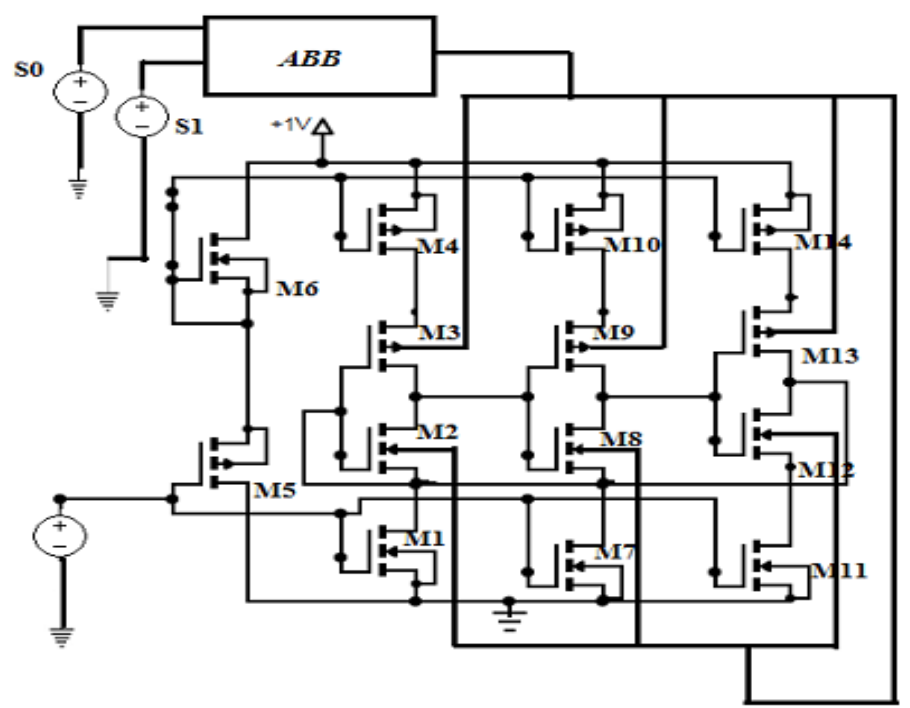

Fig.6. Ring Oscillator with PMOS and NMOS Bulk Connected to Adaptive Body Bias

Table.1. ABB Ring Oscillator at Different Input Level

\begin{tabular}{cccccc}
\hline S0 & S1 & $\begin{array}{c}\text { Voltage } \\
\text { Level (v) }\end{array}$ & $\begin{array}{l}\text { Frequency } \\
(\mathrm{GHz})\end{array}$ & $\begin{array}{l}\text { Power } \\
(\mathrm{uW})\end{array}$ & $\begin{array}{l}\text { Phase } \\
\text { Noise } \\
(\mathrm{dB} / \mathrm{Hz})\end{array}$ \\
0 & 0 & 0.745 & 12.62 & 74.9 & -58.1 \\
0 & 1 & 0.5 & 12.56 & 132 & -58.11 \\
1 & 0 & 0.24 & 12.59 & 112 & -58.11 \\
1 & 1 & $66.4 \mathrm{u}$ & 12.54 & 197 & -58.11 \\
\hline
\end{tabular}

Oscillation frequency can further enhance by varying width of transistor, dimensions of transistor depend upon the design speciation of the oscillator, the width of inverter transistors (M4, M10, M14 at 30um) (M1, M7, M11 at 10um) (M2, M8, M12 at 120nm) (M3, M9, M13 at 240nm) the highest possible frequency achievable from this circuit is $13.7 \mathrm{GHz}$. Fig7 shows the variation in the frequency of the oscillator with width of transistor. 
This range of frequency lies to Ku band (Kurtz-Under band) primary used for satellite communication focuses on editing and broadcasting the frequency range of this band lies in $12 \mathrm{GHz}$ to $18 \mathrm{GHz}$. Variation in the Channel length results in drastic reduction in frequency by increasing the value of length, so it is advisable to avoid variations with length of MOS. Adaptive body bias circuit with 3 stage current starved ring oscillator present phase noise $-58.11 \mathrm{db} / \mathrm{Hz}$. Further improvement in phase noise is possible by varying width of MOS. Phase noise varies exponentially with respect to $\mathrm{W}$ ratio shown in fig8 with increase in $\mathrm{W}$ ratio of transistor, phase noise effect is better which is always desirable.

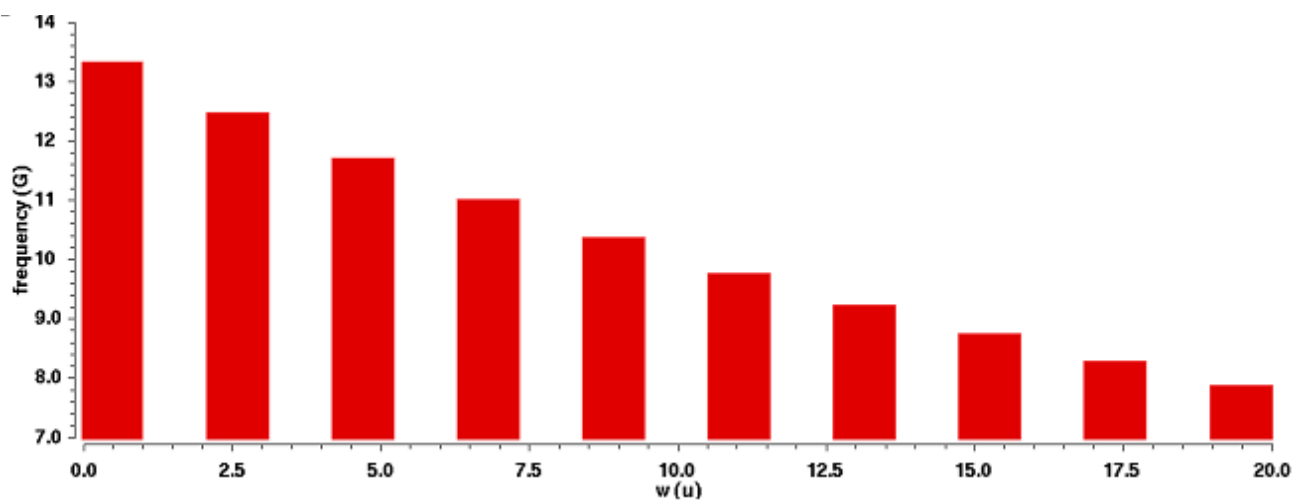

Fig7. Variation of Frequency with respect to W/L Ratio of ABB Ring Oscillator

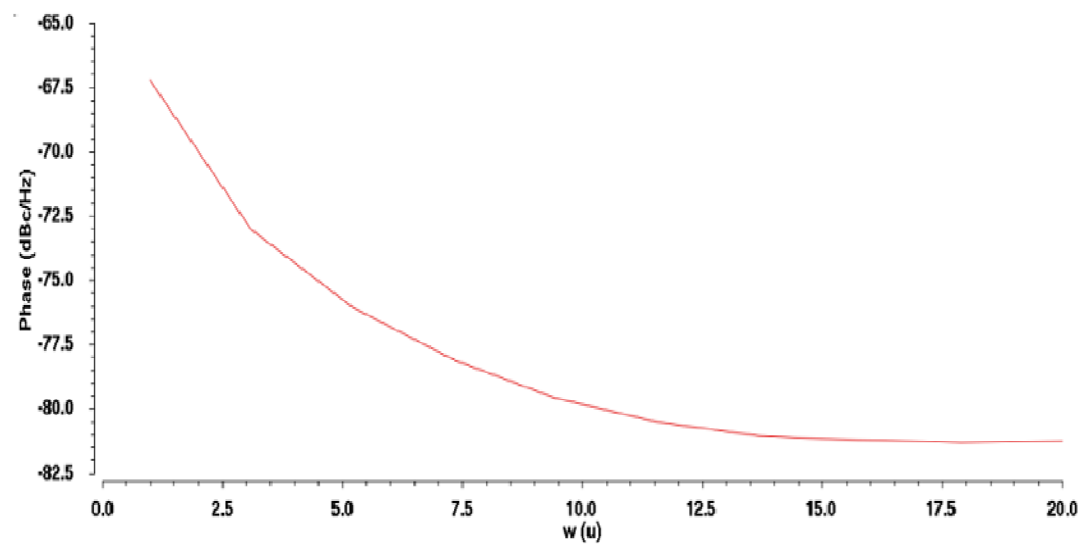

Fig.8. Phase Noise of ABB CS oscillator vs W/L ratio

Table.2. Comparative study of Ring Oscillator with Body Bias

\begin{tabular}{|c|c|c|c|c|c|c|c|c|c|c|c|}
\hline \multirow[b]{2}{*}{ Reference } & \multirow[b]{2}{*}{ Process } & \multirow[b]{2}{*}{ Type } & \multicolumn{4}{|c|}{ Operating Frequency with body boas $0.5 \mathrm{v}$} & \multicolumn{4}{|c|}{ Power } & \multirow[b]{2}{*}{$\begin{array}{l}\text { Phase Noise } \\
(\mathrm{dB} / \mathrm{Hz})\end{array}$} \\
\hline & & & Without Bias & $\begin{array}{c}\text { PMOS } \\
\text { Reverse Bias }\end{array}$ & $\begin{array}{c}\text { NMOS } \\
\text { Reverse Bias }\end{array}$ & Adaptive Bias & Without Bias & $\begin{array}{c}\text { PMOS } \\
\text { Reverse Bias }\end{array}$ & $\begin{array}{c}\text { NMOS } \\
\text { Reverse Bias }\end{array}$ & Adaptive Bias & \\
\hline [7] & CMOS180nm & Ring & $\begin{array}{c}0.958 \mathrm{G}- \\
4.46 \mathrm{G}\end{array}$ & & & & $0.226 \mathrm{u}$ & & & & -94.51 \\
\hline [13] & CMOS45nm & Ring & & $5.33 \mathrm{G}$ & $3.8 \mathrm{G}$ & $4.76 \mathrm{G}$ & & $2.68 \mathrm{u}$ & $5.68 \mathrm{u}$ & $12.03 \mathrm{n}$ & \\
\hline [15] & CMOS45nm & Ring & $5.63 \mathrm{G}$ & & & & $10.15 \mathrm{u}$ & & & & -6400 \\
\hline This Work & CMOS90nm & Current Starve & $0.626 \mathrm{G}$ & $0.5 \mathrm{G}-10.1 \mathrm{G}$ & $0.5 \mathrm{G}-12.5 \mathrm{G}$ & $\begin{array}{l}10.063 \mathrm{G}- \\
10.066 \mathrm{G}\end{array}$ & $490 \mathrm{u}$ & $30 \mathrm{u}$ & $250 \mathrm{u}$ & $2.21 \mathrm{~m}$ & -60 \\
\hline
\end{tabular}




\section{Conclusion}

A Body biasing is alternative method to increase the oscillation frequency of ring oscillator. A 3 stage current starve VCO without body bias can have oscillation frequency only $626 \mathrm{MHz}$ suitable for L-band application; reverse biasing the PMOS bulk terminal can achieve the oscillation frequency is $500 \mathrm{MHz}-$ $10.01 \mathrm{GHz}$ for control voltage $0-1 \mathrm{~V}$ while NMOS biasing the achieved results are $500 \mathrm{MHz}-12.5 \mathrm{GHz}$ suitable for $\mathrm{Ku}$ band application. Table2 summarized the performance of ring oscillator with either PMOS or NMOS bulk terminal are reverse biased. Adaptive body bias network provides bias voltage for body terminal allow the user to switch reverse bias. PMOS body bias result in less power dissipation compare to NMOS, adaptive bias network yield sustains high oscillation frequency.

\section{References}

[1] Behazd Razavi, "Design of analog cmos integrated ciruit", July 2000.

[2] Eitenne sicard, Sonia Delmas "Advanced CMOS cell Design "Tata McGraw Hill Professional, 2007

[3] P. M. Farahabadi, H. Miar-Naimi, A. Ebrahimzadeh, "A New Solution to Analysis of CMOS Ring Oscillators", Iranian Journal of Electrical \& Electronic Engineering, vol. 5, no. 1, March 2009.

[4] J.K.Panigrahi, D.P.Acharya, "Performance Analysis and Design of Wideband CMOS Voltage Controlled Ring Oscillator", IEEE $5^{\text {th }}$ International Conference on Industrial and Information Systems Proceedings, pp 234-238, Jul29-Aug 01, 2010.

[5] M K Mandal, B C Sarkar," Ring oscillators: Characteristics and applications" Indian Journal of pure and applied physics Vol.48,pp136-145,2010.

[6] M K Mandal, B C Sarkar, "Ring oscillator: Characteristics and application", Indian journal of pure \& applied physics, vol. 48, pp. 136-145, February 2010.

[7] Ashish Raman and R.K sarin "1P6M 0.18- $\mu$ n Low Power CMOS Ring Oscillator For Radio Frequency Application "International journal of computer Theory and Engineering ,Vol 3,No.6 pp770-774,2011.

[8] Sushil Kumar, Gurjt Kumar, "Design and performance of nine stage cmos based ring oscillator", International Journal of VLSI design \& Communication Systems (VLSICS), vol. 3, no. 3, June 2012

[9] Sushil Kumar and Dr. Gurjit Kaur, "Design and performance analysis of nine stages CMOS based ring oscillator," International Journal Of VLSI Design \& Communication Systems Vol.3, No.3, 2012.

[10] X. Gui, M. M. Green, "Design of CML Ring Oscillators With Low Supply Sensitivity", IEEE Transactions on Circuits and Systems I: Regular Papers, vol. 60, no. 7, pp. 1753-1763, July 2013.

[11] Abhishek Kumar. "Effect of Body Biasing Over CMOS Inverter" International Journal of electronics \& communication technology, Vol 4, issue 1, pp 369-371, Jan-march 2013.

[12] Vandna Sikarwar, Neha Yadav, Shyam Akashe," Design and analysis of CMOS ring oscillator using 45 nm technology" IEEE 3rd International conference on Advance computing, pp1491-1495, 2013

[13] Akansha SHRIVASTAVA, Anshul SAXENA, Shyam AKASHE "High performance of low voltage controlled ring oscillator with reverse body biasing" Frontier of optoelectronics, Vol.6,Issue3 pp-338-345, 2013

[14] Abbas Ramazani, Sadegh Biabani, Gholamreza Hadidi, "CMOS ring oscillator with combined delay stages", International ournal ofelectronics and Communication. (AEU), vol. 68, pp. 515-519, 2014.

[15] Kriti Tiwari, Abhishek Kumar. "11 GHz CMOS Ring oscillator" International Conference on Computing, Communication, and Automation", pp 1280-1283, 2015 


\section{Authors' Profiles}

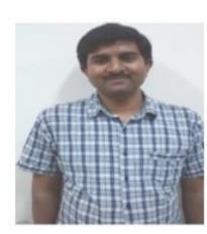

Abhishek Kumar pursuing $\mathrm{PhD}$ in Electronics and Electrical Engineering from Lovely Professional University, Punjab, India His area of interest is secured hardware design for random number and secret key, additional silicon IC based feature included in cryptographic module. He is focused on CMOS circuit design for low power, data converter, memory unit and computational circuit at CMOS 90nm technology.

Kriti Tiwari Have completed B.Tech \& M.Tech(Electronics \& Communication Engg) from Lovely Professional University, Punjab, India. Her research interest includes high frequency oscillator design with CMOS technology.

How to cite this paper: Abhishek Kumar, Kriti Tiwari,"Wide Tuning Range CMOS VCO", International Journal of Engineering and Manufacturing(IJEM), Vol.7, No.5, pp.31-38, 2017.DOI: 10.5815/ijem.2017.05.03 